ReVista de La Escuela de CIENCIAS DE LA EduCACIÓN, AÑo 17, NRO. 16, VOL. 2, JULIO A DiCIEMBRE DE 2021. PÁGINAS 135-144. ISSN 2362-3349 (EN LÍNEA). COMUNIDADES DE APRENDIZAJE: UNA EXPERIENCIA EN Y PARA LA PROFESIONALIZACIÓN DOCENTE EN LA UNIVERSIDAD NACIONAL DE EDUCACIÓNECUADOR. VÍCTOR MIGUEL SUMBA ARÉVALO. JOHANNA GRACE MEJÍA VERA.

COMUNIDADES DE APRENDIZAJE:

\title{
UNA EXPERIENCIA EN Y PARA LA PROFESIONALIZACIÓN DOCENTE EN LA UNIVERSIDAD NACIONAL DE EDUCACIÓN-ECUADOR
}

\author{
Víctor Miguel Sumba Arévalo* \\ Escuela de Educación Básica Luis Cordero Crespo, Ecuador \\ vimisuare@yahoo.es \\ Johanna Grace Mejía Vera* \\ Escuela de Educación Básica Federico Proaño, Ecuador \\ johannamejiaec@yahoo.com
}

Recibido: 30/06/2020 - Aceptado: 14/12/2020

\section{Resumen}

El presente artículo presenta la experiencia-reflexión de la puesta en marcha de las Comunidades de Aprendizaje como parte del proceso formativo de los estudiantes-docentes del séptimo ciclo de las carreras de Educación Básica y Educación Intercultural Bilingüe, modalidad a distancia del Plan para la Profesionalización docente de la Universidad Nacional de Educación (UNAE) durante el ciclo académico abril-agosto 2019. Para responder al núcleo problemático ¿cómo participa la comunidad educativa? desde el ámbito de las prácticas preprofesionales apunta a la conformación de dichas comunidades considerándolas como espacios para la gestión del conocimiento a través de la investigación acción participativa y la perspectiva del aprendizaje dialógico para generar la reflexión en, sobre y para la práctica. Este análisis de procesos orienta a describir la participación de los estudiantes-docentes en la Comunidad de Aprendizaje para la gestión del conocimiento, considerando a cada uno de ellos como portadores de saberes, de esta manera se garantiza la participación igualitaria de los actores educativos que se comprometen a la transformación social. Todo ello, nos lleva a considerar a las Comunidades de Aprendizaje como espacios de interaprendizaje que fomentan la reflexión y construcción de nuevos aprendizajes en y para la profesionalización docente.

Palabras clave: Comunidad de Aprendizaje - Aprendizaje dialógico - Profesionalización docente Gestión del conocimiento y Transformación social.

\footnotetext{
* Profesor de Educación Básica (ISPED Luis Cordero-Ecuador), Licenciado en Educación General Básica (Universidad de Cuenca-Ecuador), Máster en Psicopedagogía y Máster en Neuropsicología y Educación (Universidad Internacional de la Rioja-España), Doctorando en Educación (Universidad Nacional de Rosario-Argentina). Ha desempeñado la función de Docente Tutor en la Universidad Nacional de Educación (Azogues-Ecuador) desde marzo 2018 a marzo 2020. Actualmente docente fiscal en la Escuela "Luis Cordero Crespo" Cuenca-Ecuador. Co-investigador en el Proyecto: "Néopass@ction@: una experiencia colaborativa de video-formación para la profesionalización docente en Ecuador" de la Universidad Nacional de Educación-UNAE.

** Licenciada en Psicología Educativa (Universidad de Cuenca - Ecuador), Máster en Métodos de Enseñanza en Educación Personalizada (Universidad Internacional de la Rioja - España), ex Docente Tutora de Universidad Nacional de Educación, en la actualidad Docente en Educación Básica en la Escuela "Federico Proaño". Ha desempeñado cargos directivos teniendo bajo su cargo el manejo de la pedagogía de los centros educativos, al igual que el desarrollo de procesos de capacitación docente. Co-investigadora en el Proyecto "Néopass@ction@: una experiencia colaborativa de video-formación para la profesionalización docente en Ecuador" de la Universidad Nacional de Educación-UNAE.
} 


\title{
LEARNING COMMUNITIES:
}

\section{AN EXPERIENCE IN AND FOR TEACHING PROFESSIONALIZATION AT THE NATIONAL UNIVERSITY OF EDUCATION-ECUADOR}

\begin{abstract}
This article presents the experience-reflation in the implementation of the learning communities as part of the training process of the students-teachers in the seventh cycle of the careers of Basic Education and Bilingual Intercultural Education, a distance modality of the Plan for Professionalization teacher at the Universidad Nacional de Educación (UNAE) during the academic year April-August 2019. To respond to the problem nucleus, how does the educational community participate? Through the field the objective of pre professional practices, it aims at the conformation of these communities considering as spaces for knowledge management through participatory action research and the perspective of dialogical learning to generate in and about reflection practice. This process analysis aims to describe the participation of teachers-students in the Learning Community for knowledge management, considering each of the teachers as knowledge carriers, thus ensuring the equal participation of the educational actors involved. They commit to social transformation. All this leads us to consider learning communities as inter-learning environments that promote reflection and the construction of the new learning in and for teacher professionalization.
\end{abstract}

Keywords: Learning community - Dialogical learning - Teacher professionalization - Knowledge management and social transformation.

\section{Introducción}

La transformación en educación exige un esfuerzo mancomunado entre todos los actores educativos, considerar al otro como portador de conocimientos, habilidades, experiencias, creencias, etc. contribuye en la construcción y consolidación de planes y procesos en beneficio de la educación. Los procesos de enseñanza y de aprendizaje no dependen solo de lo que sucede en el aula, sino también de lo que ocurre en otros espacios como en el hogar, comunidad, amigos, etc. Como indica Botton (2015) el aprendizaje es el resultado de la cadena de interacciones con diversas personas y contextos.

De ahí que el tema e interés de este artículo es presentar a las Comunidades de Aprendizaje como un espacio y tiempo para mejorar la calidad de la educación mediante la construcción y ejecución de propuestas con la interacción activa, participativa e igualitaria de docentes, directivos, estudiantes, padres de familia, miembros del entorno, entre otros. Consideramos además, que la propia interacción genera debate y co-construcción de significados los que configuran o reconfiguran nuevos aprendizajes en sus miembros. También, dicha Comunidad de Aprendizaje constituye una herramienta para la formación permanente del profesorado, pues requiere que los miembros que la conforman sean investigadores críticos y reflexivos de su propia práctica (Alcedo et. al., 2014).

En tal sentido, en el presente texto se pretende describir la participación de los estudiantesdocentes en las Comunidades de Aprendizaje para la gestión del conocimiento como parte de su proceso de formación en la UNAE (Azogues-Ecuador). El tratamiento teórico y metodológico de las Comunidades de Aprendizaje se desarrolla en los módulos respectivos del séptimo ciclo, mientras que la puesta en marcha se orienta y desarrolla en la asignatura de Cátedra Integradora con el acompañamiento del docente autor ${ }^{1}$ $\mathrm{y}$ tutor ${ }^{2}$.

Cabe mencionar que las carreras de Educación Básica y Educación Intercultural Bilingüe, modalidad a distancia del Plan para la Profesionalización docente responde a la necesidad de formar a docentes en ejercicio que no poseen título de tercer nivel, cuentan con una experiencia mínima de cinco años, desempeñan sus funciones en nivel de Educación General Básica, edad comprendida entre treinta y cincuenta años y no se encuentran matriculados en otra institución de Educación Superior (Vilanova, et. al., 2017). Actualmente dichas carreras son desarrolladas en diez de las veinticuatro provincias del Ecuador.

\section{Marco Teórico: las Comunidades de Aprendizaje}

A continuación, presentamos una revisión de los principales representantes y exponentes de las Comunidades de Aprendizaje, así como también expondremos algunas experiencias de su aplicación.

Para Flecha (2011) la Comunidad de Aprendizaje constituye; una comunidad de personas que aprenden conjuntamente unas de otras [...] son escuelas, centros, colegios, institutos que deciden funcionar como punto de aprendizaje, con lo cual pierden ese carácter anterior jerárquico [...] aprenden entre ellos y

\footnotetext{
${ }^{1}$ De acuerdo al Modelo de Prácticas de las carreras a distancia el docente autor de Cátedra Integradora coordina la contribución de las asignaturas para mejorar la práctica, además, crea y orienta la reflexión sobre la práctica.

${ }^{2}$ De acuerdo al Modelo de Prácticas para las carreras a distancia, el docente tutor acompaña al estudiante en el aula, escuela o contexto escolar, acompaña en el proceso de reflexión e investigación de manera articulada con los demás actores.
} 
ReVista de La Escuela de CIENCIAS DE LA EduCACIÓN, AÑo 17, NRO. 16, VOL. 2, JULIO A DiCIEMBRE DE 2021. PÁGINAS 135-144. ISSN 2362-3349 (EN LÍNEA). COMUNIDADES DE APRENDIZAJE: UNA EXPERIENCIA EN Y PARA LA PROFESIONALIZACIÓN DOCENTE EN LA UNIVERSIDAD NACIONAL DE EDUCACIÓNECUADOR. VÍCTOR Miguel SUMBA ARÉVALO. JOHANNA GRACE MEJÍA VERA.

entre ellas, conjuntamente profesorado, familiares, niños y niñas presencialmente y también a través de internet.

Citando a Beltrán, et. al. (2015) las Comunidades de Aprendizaje "son proyectos de transformación social y cultural de un centro educativo y de su entorno, basados en el aprendizaje dialógico, en espacios concretos, incluyendo el aula de clases" (Beltrán, 2015, p.59). Además mencionan sus características fundamentales: 1) brindan gran valor a la inteligencia cultural de los adultos, 2) transforman el contexto en el que se desenvuelven, no buscan la adaptación, 3) su aprendizaje pedagógico se basa en el cooperativismo y el aprendizaje dialógico y, 4) genera prácticas inclusivas, donde todos tienen algo que enseñar. A esta última característica, Trujillo et. al. (2015) añaden la idea de que todos ignoramos y sabemos algo, por lo que es necesario establecer redes de aprendizaje interconectadas de tal manera que nos permitan aprender y gestionar el conocimiento, a su vez permiten que cada individuo descubra el potencial de lo que puede enseñar.

Cadena y Orcasitas (2016) acotan que las Comunidades de Aprendizaje constituyen planes de transformación social y cultural en las instituciones educativas enfocadas en la superación del fracaso escolar y la superación de conflictos teniendo como base el aprendizaje dialógico, una convivencia democrática y solidaria. Hacen énfasis en que el diálogo debe ser constante entre todos los participantes, no se debe delimitar a reuniones esporádicas. Los beneficios de la aplicación de las Comunidades de Aprendizaje son varios, a continuación, se mencionan algunos de ellos:

- Permite el involucramiento de la familia, más allá del rol convencional que tradicionalmente vienen cumpliendo (seguimiento y control), dando paso a extensiones de nuevos contextos de aprendizaje, pues en el hogar también participan en la realización de las actividades de aprendizaje de los estudiantes, adoptando la figura de educadores.

- La conformación de las Comunidades de Aprendizaje entre profesores con un mismo objetivo genera un sentimiento compartido de responsabilidad, respeto y trabajo, aportando soluciones necesarias que requiera la comunidad educativa.

- Promueve la reflexión sobre la práctica, no de manera individual, sino como una práctica pedagógica colaborativa dando lugar a la construcción de un nuevo conocimiento, fruto de una construcción colectiva, donde todos tienen algo que aprender y mucho que enseñar (Alcedo et. al., 2014).

- Ayuda a trabajar en la inclusión educativa y redes de trabajo, donde no únicamente recae la responsabilidad sobre profesionales especializados, sino sobre una red comunitaria, dentro y fuera de la escuela (Molina, 2015).

Cantero y Pantoja (2016) acotan que las Comunidades de Aprendizaje han sido parte de las transformaciones en los centros educativos de la provincia de Jaén en España, donde se han podido observar actuaciones inclusivas; pues parten de las potencialidades de los individuos y no de las dificultades que presentan. Permite también el cierre de brechas de desigualdades y participación al no requerir de grandes recursos, más bien aprovechar la predisposición de sus miembros y el trabajo mancomunado. Las Comunidades de Aprendizaje permiten procesos que llevan a transformaciones educativas, a través de características en común que poseen como: liderazgo distribuido, valores, visión, confianza, respeto y responsabilidad compartida (Krichesky y Murillo, 2011).

Por otro lado, Molina (2015) indica que la aplicación de las Comunidades de Aprendizaje mejora los resultados académicos y la convivencia entre sus miembros. También Beltrán et. al. (2015) consideran que la aplicación de las Comunidades de Aprendizaje en el Colegio Integrado Yarima (Colombia) fue un factor influyente para evidenciar que en ese período, los estudiantes aumentaron su puntaje en las pruebas "Saber".

De acuerdo a Jiménez y Rodríguez (2016), la puesta en marcha de las Comunidades de Aprendizaje implica cinco fases:

Fase de sensibilización. Se reúnen los actores y/o invitados con quienes se conformará la Comunidad de Aprendizaje y se comparten las fortalezas, necesidades, problemas o inquietudes educativas evidentes o no en la práctica docente. Con ello se resalta la necesidad de unificar esfuerzos para solventarlos. Se pueden relatar experiencias de otras instituciones y los resultados de su aplicación.

Fase de toma de decisión. A través del diálogo igualitario y democrático se llega a la determinación sobre la conformación de la Comunidad de Aprendizaje.

Fase de sueño. Es el inicio de la transformación educativa, en donde todos los actores, en base a sus aspiraciones, anhelos y deseos plasman con creatividad el tipo de escuela ideal.

Fase de selección de prioridades. Se hace un contraste entre lo que se estableció en la fase de sueños y lo que es pertinente, necesario y prioritario para dar solución a la problemática fijada. Se analiza con qué tipos de recursos, materiales y personas se cuenta para poder continuar con el proceso de transformación.

Fase de planificación. Se inicia con la cristalización de los sueños, fijando actividades, plazos y responsabilidades compartidas. Este proceso se lleva a cabo a través de reuniones periódicas tipo asambleas donde todas las voces son importantes.

Jiménez y Rodríguez (2016) consideran importante que se efectúen acciones que permitan la sostenibilidad de las Comunidades de Aprendizaje, proponen una etapa investigativa sobre la propia experiencia de la participación de los diferentes actores; otra de consolidación por grupos de interés o 
ReVista de La Escuela de CIENCIAS DE LA EduCACIÓN, AÑo 17, NRO. 16, VOL. 2, JULIO A DiCIEMBRE DE 2021. PÁGINAS 135-144. ISSN 2362-3349 (EN LÍNEA). COMUNIDADES DE APRENDIZAJE: UNA EXPERIENCIA EN Y PARA LA PROFESIONALIZACIÓN DOCENTE EN LA UNIVERSIDAD NACIONAL DE EDUCACIÓNECUADOR. VÍCTOR Miguel SUMBA ARÉVALO. JOHANNA GRACE MEJÍA VERA.

trabajo que permitirá dar paso a la siguiente etapa, que es la de evaluación de todo el proceso con los resultados conseguidos.

Por otro lado, Morales et. al. (2013) exponen los tipos de Comunidades de Aprendizaje según la agrupación de los actores, quienes desde su experiencia, formación, interacción, o pertenencia a un sector contribuirán a emprender acciones en beneficio de la educación.

Comunidades Profesionales de Aprendizaje (CPA). Caracterizada por el agrupamiento de docentes profesionales, que no necesariamente deben ser de la misma área o institución, es suficiente compartir una problemática, interés u objetivo en común. Estas comunidades, permiten el fortalecimiento del sentido de pertenencia al centro educativo, a la vez que se considera una estrategia formativa.

Comunidades Áulicas. Ocurre dentro del salón de clase con la participación del docente, estudiantes y la colaboración de agentes externos como padres de familia, profesionales y especialistas. El docente cambia su rol, siendo aquel que promueve la participación desde el aporte de todos los actores, para solucionar la problemática que se haya fijado.

Comunidades Institucionales. Agrupación de instituciones con la misión de gestionar nuevas culturas escolares, generando nuevos principios con miras a la innovación, equidad y calidad en la educación. Se aprovechan todas las fortalezas y potencialidades que cada centro posee, generando redes de trabajo y alianzas estratégicas.

Comunidades Virtuales. Debido a la proliferación de la tecnología y la amplia cobertura que proporciona el ciberespacio es posible generar sitios que permitan el debate en torno a una temática específica, permitiendo que una misma persona se desenvuelva en diferentes roles como autor, crítico o simplemente sea el lector de lo expresado.

De ahí, que es necesario que dentro de las Comunidades de Aprendizaje todos los actores estén convencidos de su participación y el valioso aporte en este proceso. El liderazgo compartido es un elemento clave para fortalecer al grupo.

\section{El Aprendizaje Colaborativo en Comunidades de Aprendizaje}

Uno de los requisitos para generar el desarrollo de una institución constituye el trabajo colaborativo y por ende el compromiso compartido entre quienes la conforman. En el sistema educativo, dicho trabajo se vuelve cada vez más necesario para diagnosticar y responder a las necesidades, demandas y problemas de la institución educativa. El aprendizaje colaborativo para Herrera y Guevara (2019) es entendido como el proceso en el cual, cada persona aporta desde su experiencia personal, profesional, puntos de vista, opiniones, etc. con el propósito de ayudar a sus compañeros. En tal sentido, para generar resultados favorables, se debe partir desde el fortalecimiento de las relaciones interpersonales para que cada docente se sienta apoyado por el otro.

Desde la propia experiencia como docentes hemos podido evidenciar que cada profesor puede considerarse como un mundo separado, cada uno conoce las necesidades del aula y las trata de resolver por sí solo. Es así como se hace evidente la importancia de generar espacios de interacción y fortalecimiento de las capacidades docentes. En las carreras a distancia de la UNAE, se propicia el desarrollo del trabajo y aprendizaje colaborativo con la finalidad de compartir los conocimientos que tiene cada docente. A decir de Torres "La cooperación y la solidaridad deben ser vistos como norma y como recurso no únicamente para tareas de la supervivencia sino para asegurar los conocimientos, habilidades, valores y actitudes que forman parte del fondo cultural y educativo de una comunidad" (2004, p. 6). Esta estrategia guía desde la reflexión conjunta de las prácticas pedagógicas tradicionales hacia nuevas formas de enseñar y aprender, es decir, conlleva a desaprender y reaprender.

Las Comunidades de Aprendizaje aportan de manera significativa frente a otros tipos de propuestas colaborativas, principalmente en el punto que centran su objetivo de estudio y cambio. A continuación, haremos un contraste con otras acciones formativas tales como: Talleres de Educadores, Aprendizaje Situado y Ateneos Docentes.

Los Talleres de Educadores, apuntan a la modificación de las relaciones pedagógicas y las prácticas del docente a partir del cuestionamiento y reflexión del saber pedagógico, es decir ¿por qué lleva a cabo su práctica de esa manera?, ¿Es producto de la experiencia vivida como estudiante? Así entonces ellos se convierten en protagonistas e investigadores de su práctica, asumiendo el rol de estudiantes, lo que da paso a la construcción de un nuevo conocimiento. Esto se realiza en un marco colaborativo y democrático con otros docentes (Assael y Guzmán, 1996).

Si bien es cierto que las Comunidades Profesionales de Aprendizaje al igual que los Talleres de Educadores trabajan con docentes en grupos pequeños, sin embargo, centran sus diálogos en cómo asegurar que los estudiantes aprendan, siendo entonces el punto de partida las necesidades de los estudiantes y esto es lo que marcará los temas de formación de los docentes. No obstante, esto no queda únicamente en un conocimiento recibido, sino que se convierte en una práctica de innovación que luego de su implementación es evaluada, estableciendo un nuevo ciclo de mejora (Krichesky y Torrecilla, 2011).

A estas dos concepciones, viene a sumar el Aprendizaje Situado, que entre sus postulados principales señala que las narrativas que se realicen de los procesos vividos. Deben ser analizadas a profundidad a través de una lectura crítica, que permita conocer el porqué de su actuar, que no sea una efímera evocación de hechos, sino que permitan crear un saber compartido en medio de la gran diversidad. Lo anterior lleva a un proceso de transformación, sobre la base de la comprensión de lo que ocurre en la cotidianidad (De Antonio y Gonçalves, 2017). 
ReVista de La Escuela de CIENCIAS DE LA EduCACIÓN, AÑo 17, NRO. 16, VOL. 2, JULIO A DiCIEMBRE DE 2021. PÁGINAS 135-144. ISSN 2362-3349 (EN LÍNEA). COMUNIDADES DE APRENDIZAJE: UNA EXPERIENCIA EN Y PARA LA PROFESIONALIZACIÓN DOCENTE EN LA UNIVERSIDAD NACIONAL DE EDUCACIÓNECUADOR. VÍCTOR Miguel SUMBA ARÉVALO. JOHANNA GRACE MEJÍA VERA.

Finalmente, los Ateneos Docentes, han sido pensados para la formación de los docentes noveles frente a las problemáticas particulares que tienen que enfrentar en las aulas (Alen, 2013). Esto, a diferencia de la CPA se desarrolla a través de pares, donde el coordinador es quien guía el proceso narrativo del docente a través de diarios de campo. El punto de sinergia que tienen con las CPA es que están conscientes de que no sirve únicamente la reflexión de las prácticas, sino se debe establecer una planificación de cambio para que de esta manera surja efecto en la práctica.

En definitiva, y desde el punto de vista de los investigadores, las Comunidades de Aprendizaje permiten la intervención e interacción de otros agentes, no únicamente de docentes. Esta conformación permite tener mayores posibilidades de comprender y solucionar problemas referidos a la educación. Consideramos a éstas como espacios propicios para la construcción y reconstrucción de saberes, conocimientos, experiencias, habilidades en los diferentes miembros de la comunidad educativa.

\section{Aprendizaje Dialógico en Comunidades de Aprendizaje}

Aubert et. al. (2009) mencionan que los fundamentos para la consolidación del aprendizaje dialógico tienen sus bases en la propuesta de Vygotsky (1996) quien resalta la importancia y la necesidad de la interacción social para el aprendizaje; Freire (1970) quien marca la naturaleza dialógica de la persona; y de Bruner (1996), Rogoff (1993) y Wells (2001) que señalan la necesidad de convertir el diálogo en un eje clave del proceso de enseñanza y de aprendizaje.

Por tal razón, cuando el diálogo es la base para consensuar cambios y lograr una transformación individual y social, se hace referencia al aprendizaje dialógico (Castro et. al., 2014). Desde el enfoque comunicativo, el aprendizaje dialógico se concibe como el aprendizaje basado en la interacción con otras personas. En tal sentido, el diálogo, palabra, discurso con el otro se convierten en instrumentos para construir significados y reconocer el conocimiento cultural de las personas; es decir, se considera que todas las personas tenemos conocimiento para aportar (Comunidades de Aprendizaje, s.f.). Entonces, las características para generar un aprendizaje dialógico son las relaciones democráticas, horizontales, igualitarias sin que existan relaciones de opresión de unos sobre otros.

Por otro lado, Valls y Munte (2010) señalan que el éxito de las comunidades de aprendizaje a través del aprendizaje dialógico se debe a ciertas características claves:

Principio de transformación. Es decir, no se centra en la queja o el déficit de algo, sino más bien, el aprendizaje dialógico parte de la inteligencia cultural de los estudiantes y todos aquellos que lo rodean.

Inteligencia cultural. Está conformada por la inteligencia, práctica académica y comunicativa.

Principio de dimensión instrumental. Todos los objetivos y actividades que se plasmen deben tener el carácter de inclusivo, debe servir para que todas las personas aprendan más. Este principio es visibilizado en la fase de sueños, donde todos expresan sus deseos e ideales.

Solidaridad. Se plantea un objetivo en común y entre todos los miembros se apoyan para aprender, dejando atrás el espíritu competitivo, creando más bien el espacio de análisis de posibles orígenes de las desigualdades sociales.

Diálogo igualitario. Se parte de una necesidad de la comunidad, donde a través de consensos y aportes de sus miembros se toman las mejores decisiones. La responsabilidad de qué y cómo aprender ya no recae únicamente en el profesional, es ahora una decisión abierta de todos.

Creación de sentido. Aubert et. al. (2009) indican que "no se refiere únicamente al sentido que damos a los aprendizajes, sino que es un concepto mucho más amplio y, del mismo modo que los significados se crean en relación con las otras personas" (p. 136).

Igualdad de diferencias. Todos pueden dar aportes valiosos e interesantes. Así por ejemplo, los estudiantes con necesidades educativas especiales se ven doblemente favorecidos, mayor acceso al currículo y el fortalecimiento de su autoestima al dar su aporte a la comunidad (Álvarez et. al., 2013).

Los beneficios de la implementación del aprendizaje dialógico en las Comunidades de Aprendizaje, según Domínguez (2018) son: disminución del absentismo escolar, mejora de la convivencia de todos los miembros, aumento del rendimiento académico y fomento de prácticas inclusivas. Adicional a ello, la creación de las Comunidades de Aprendizaje fortalece el proceso de formación de los docentes, ya que no sólo enseñan, también aprenden de manera conjunta. Los aprendizajes traspasan las paredes del aula y se abren a nuevos escenarios inmediatos propios de la comunidad. Incluso se forman en aspectos no contemplados en su formación inicial, por ejemplo siendo gestores culturales de transformaciones sociales cercanas, entre otros (Soria y Hernández, 2017).

Para generar aprendizajes entre docentes y reforzar la práctica pedagógica, uno de los aspectos a considerar es el cúmulo de conocimientos teóricos y prácticos que tiene cada docente con base a sus experiencias. El compartir saberes (saber y saber hacer), genera nuevos aprendizajes mediante la correflexión y el aprendizaje igualitario, rompiendo de esta manera la cultura balcanizada que impregna a la mayoría de las prácticas docentes, aprovechando al máximo todos los recursos físicos y humanos con los que cuenta la comunidad (Domínguez, 2018).

La formación de los docentes en las Comunidades de Aprendizaje parte de relaciones igualitarias, donde todos tienen algo que aprender y también algo que enseñar. Por tal motivo, lo que se pretende desde este punto de formación permanente es la difusión de cada experiencia docente, a esto se articula el debate de saberes disciplinares que sustentan estas experiencias. El diálogo, trabajo colaborativo, discusión y análisis crítico reflexivo permite que los docentes en la comunidad generen procesos de transformación social desde su contexto (Salamanca et. al., 2014). 
ReVista de La Escuela de CIENCIAS DE LA EduCACIÓN, AÑo 17, NRO. 16, VOL. 2, JULIO A DiCIEMBRE DE 2021. PÁGINAS 135-144. ISSN 2362-3349 (EN LÍNEA). COMUNIDADES DE APRENDIZAJE: UNA EXPERIENCIA EN Y PARA LA PROFESIONALIZACIÓN DOCENTE EN LA UNIVERSIDAD NACIONAL DE EDUCACIÓNECUADOR. VÍCTOR Miguel SUMBA ARÉVALO. JOHANNA GRACE MEJÍA VERA.

\section{Proceso metodológico y didáctico en Comunidades de Aprendizaje}

Este trabajo se enmarca dentro del campo de la investigación cualitativa en la que por medio de la observación se han registrado los datos, y la interpretación de los mismos nos lleva a conocer y comprender (García y Giacobbe, 2009) la participación de los docentes en la generación de conocimientos.

La información para la interpretación se obtiene de manera directa de cada proceso de consolidación de las Comunidades de Aprendizaje. Si bien es cierto que la propuesta se desarrolla con todos los estudiantes-docentes del séptimo ciclo (durante el periodo abril-agosto 2019) de las carreras a distancia de la UNAE, para el desarrollo de este estudio se consideran cuatro grupos (que posteriormente se constituyen en Comunidades de Aprendizaje), de los cuales, dos grupos pertenecen a la provincia del Azuay y dos de la provincia del Cañar-Ecuador.

La observación directa (diario de campo) y registro en video del proceso diagnóstico y ejecución de la actuación educativa de éxito permite identificar el comportamiento de cada uno de los estudiantesdocentes participantes. El seguimiento constante (registro documental) mediante la plataforma y encuentros presenciales facilita seguir la ruta en la construcción de la comunidad, pero sobre todo la actuación para responder a la problemática. Al finalizar el ciclo académico, mediante un grupo de discusión (grabación en video) se conoce el valor que cada docente asigna a la funcionalidad de la Comunidad de Aprendizaje y el aporte a su formación. Para el proceso de análisis de la información se establecen categorías de análisis: participación docente, gestión de conocimientos, funcionalidad y valor de las comunidades, y, análisis/reflexión de la práctica docente. Con base al análisis se procede a la interpretación que conlleva a interrelacionar varias categorías para comprender el aporte de la Comunidades de Aprendizaje en la profesionalización docente.

Otra fuente de información que permite contrastar, a la vez que triangular la información corresponde al informe del proyecto integrador de saberes denominado PIENSA, en el cual se registra el actuar y sentir de cada Comunidad de Aprendizaje y cada participante. Como ya se mencionó, el registro de datos de las diferentes etapas y procesos permite una triangulación de fuentes de información durante la investigación. En definitiva, el trabajo de campo, la observación directa no participante, la revisión bibliográfica y la triangulación de información otorgan características de rigurosidad, credibilidad y validez de resultados de esta investigación

Cabe recordar que la asignatura, en la cual se desarrollan las Comunidades de Aprendizaje cuenta con la orientación del docente autor y tutor, siendo este último quien acompaña en territorio en las fases de ejecución de las comunidades, por lo cual, la indagación se vincula directamente con el proceso didáctico propuesto en la asignatura de Cátedra Integradora, mismo que se detalla a continuación.

\section{Comunidades de Aprendizaje en la Profesionalización Docente}

Para responder al núcleo problémico del séptimo ciclo ¿cómo participa la comunidad educativa? en la asignatura de Cátedra Integradora se propone la conformación de dichas comunidades para la gestión del conocimiento, considerando a cada docente como portador del mismo. Para la conformación de dichas comunidades entre estudiantes-docentes se considera: situación geográfica (factibilidad para reunirse), tipo de institución (unidocente, pluridocente o completas) e interés en relación con la problemática a ser desarrollada.

Considerando los aspectos antes mencionados forman grupos para discutir sobre las dificultades que evidencian en cada institución educativa. A partir de ello identifican posibles actores internos o externos a la institución educativa para conformar la Comunidad de Aprendizaje, que generalmente constituyen otros docentes y directivos de la misma $u$ otras instituciones educativas o miembros de la comunidad educativa. Posterior a ello, planifican un taller de diagnóstico participativo mediante la cartografía social. Con este instrumento las comunidades hacen explícitos los problemas presentes en las instituciones y cómo éstos se relacionan con otras necesidades, situaciones o contextos. Es evidente la preocupación de los participantes al exponer las posibles causas que inciden en la educación de los estudiantes, entre estos mencionan: bajo involucramiento de los padres, manejo de estrategias didácticas para fortalecer el aprendizaje, comportamiento de los estudiantes, motivación hacia la enseñanza y al aprendizaje, falta de recursos didácticos, entre otras. A partir de este listado cada grupo prioriza el problema que influye de manera directa en el desarrollo del proceso de enseñanza y de aprendizaje.

Con la problemática definida se constituye de manera formal la Comunidad de Aprendizaje determinando acuerdos y compromisos para fortalecer dicho proyecto. En este punto vale resaltar que, debido a las características de los participantes se constituyen las Comunidades Profesionales de Aprendizaje (CPA), misma que se caracteriza por la conformación con profesionales involucrados de forma directa en la educación. Posterior a ello, los estudiantes-docentes realizan una búsqueda documental sobre el problema para cimentar su comprensión, dicho proceso se detalla a continuación.

\section{Fundamentación de la Experiencia-Gestión de nuevos conocimientos}

Para ampliar el conocimiento sobre la problemática, los estudiantes-docentes realizan una investigación bibliográfica orientada a la construcción del estado del arte. En esta fase, la Comunidad de Aprendizaje (especialmente los estudiantes-docentes), contrastan de manera conjunta su experiencia con fundamentos teóricos, lo que permite contrastar y reflexionar entre el conocimiento proclamado y el conocimiento en uso (Pérez, 2012). La búsqueda bibliográfica contribuye a generar nuevos aprendizajes, previo a ello existe el proceso de análisis de la información, contraste (desde la experiencia u otras teorías), interiorización de los nuevos conceptos, relación con la propia práctica, etc. 
ReVista de La Escuela de CIENCIAS DE LA EduCACIÓN, AÑo 17, NRO. 16, VOL. 2, JULIO A DiCIEMBRE DE 2021. PÁGINAS 135-144. ISSN 2362-3349 (EN LÍNEA). COMUNIDADES DE APRENDIZAJE: UNA EXPERIENCIA EN Y PARA LA PROFESIONALIZACIÓN DOCENTE EN LA UNIVERSIDAD NACIONAL DE EDUCACIÓNECUADOR. VÍCTOR Miguel SUMBa ARÉVALO. JOHANNA GRACE MEJÍA Vera.

Estos procesos para la adquisición del conocimiento se complementan con el debate entre los miembros de la comunidad, considerando las interpretaciones de cada uno y valorando las aportaciones individuales. Durante el proceso de indagación, formación y práctica de los docentes, se pone de manifiesto la epistemología, con sus formas de ser, pensar, creer como persona y profesional. Una vez que el estado del arte ha permitido ampliar la comprensión del problema y relacionar con el contexto de cada Comunidad de Aprendizaje, y como parte del componente teórico-curricular de la asignatura se analizan alternativas para intervenir en dichos espacios.

\section{Alternativas para actuar en Comunidad de Aprendizaje}

La propuesta para gestionar las Comunidades de Aprendizaje consiste en la aplicación de una de las actuaciones educativas de éxito propuestas en el proyecto INCLUD-ED ${ }^{3}$ (2006-2011). Siendo mayoritariamente Comunidades Profesionales de Aprendizaje la actuación educativa de éxito que responde a esta característica es la formación dialógica del profesorado, que de acuerdo a Roca (2016) "recoge la voz de todos los agentes implicados para construir conocimiento de forma colectiva alrededor de la ciencia, situando en el centro de análisis, la práctica educativa" (p. 13). La formación dialógica del profesorado consiste en la selección, lectura y discusión de temas pedagógicos relevantes de interés para fortalecer la práctica.

En esta actuación de éxito lo que prima es el diálogo igualitario, respeto a las ideas, percepciones, conceptualizaciones personales de cada participante, etc. Para garantizar lo expuesto anteriormente, se elige un moderador de grupo, quien entre sus funciones posee la de garantizar el respeto a las opiniones (CIPPEC, 2018). De acuerdo a la problematización, cada una de las Comunidades de Aprendizaje diseña un plan para intervenir. En primer lugar los docentes seleccionan el material a ser analizado, luego comparten dicha información con los miembros de la comunidad, solicitan la lectura previa y selección de contenidos que consideren relevantes a ser discutidos en la comunidad en una fecha determinada.

El desarrollo de la formación dialógica del profesorado inicia con las orientaciones de participación por parte del moderador, posterior a ello da a conocer el objetivo del encuentro y la metodología del trabajo (formación dialógica del profesorado). Cada participante cuenta con el material estudiado, a partir de ello, el moderador realiza preguntas para iniciar con el debate, como: ¿qué se entiende por estrategia didáctica?, ¿cuáles son las estrategias que favorecen la participación activa de los estudiantes?, ¿cuáles son las prácticas desarrolladas en relación con las estrategias para la comprensión lectora?, entre otras. Durante el debate se evidencia la participación repetitiva de algunos docentes, mientras otros responden a ciertas inquietudes y una minoría se limita a la participación pasiva en el taller. En las aportaciones a la temática se pone de manifiesto la relación de la teoría con los conocimientos (praxis) generados durante la profesión docente, se hacen evidentes las reflexiones generadas durante la revisión documental en relación con las prácticas.

Con relación a lo tratado en apartados anteriores se puede manifestar la funcionalidad de las Comunidades de Aprendizaje para potenciar la práctica de los docentes, esto considerando el contraste teórico, metodológico, didáctico y pragmático de las diferentes fuentes de información, que durante y posteriormente a la discusión, se convierten en nuevos aprendizajes. Para generar dichos conocimientos de forma conjunta, es necesario concebir al otro como portador de conocimientos, emplear el diálogo igualitario para fortalecer la participación activa de los docentes. La gestión de conocimientos converge con el trabajo colaborativo, pues, es en la sociedad donde se crean y transmiten los conocimientos. No solo se trata de acceder a la información, más bien de ser capaz de autorregularse y desarrollarse profesionalmente a lo largo de la vida, mismo que constituye una de las competencias profesionales del docente UNAE.

Tenemos que desarrollar en los docentes la capacidad de trabajar en grupo y aprender cómo aprender para asumir el destino de su desarrollo profesional futuro, el cual, para ser relevante y que cuaje en la personalidad del docente, debe ser parte de las propias necesidades y del propio convencimiento. Si el docente es capaz de autorregularse y aprender cómo aprender a lo largo de toda la vida, él mismo desarrollará la capacidad necesaria para afrontar los inevitables, sustantivos y vertiginosos cambios que la era digital impone en el conocimiento y en la vida contemporánea. (UNAE, 2017, p. 18).

Además, es necesario el desarrollo de la competencia oral y escrita en los docentes con la finalidad de comunicar las ideas, pensamientos, sentimientos, creencias, etc. La comunicación oral en las comunidades de aprendizaje es fundamental para garantizar el diálogo igualitario. Adicional a ello, la sistematización de la experiencia en las Comunidades de Aprendizaje en el PIENSA ha permitido socializar el proceso, propuestas en comunidades y los primeros resultados evidenciados.

\section{Discusión en relación a la Gestión del Conocimiento en Comunidades de Aprendizaje \\ De la propuesta de conformación de las Comunidades de Aprendizaje en y para la profesionalización de docentes se puede manifestar que:}

\footnotetext{
${ }^{3}$ INCLUD-ED, fue un proyecto del Programa Marco de la Comisión Europea, realizado entre los años 2006-2011 con investigadores de 14 países europeos. Realizó el análisis de estrategias que permiten mejorar los aprendizajes en los sectores más desfavorecidos, con la participación de todos los actores. Visibilizó las acciones educativas de éxito que favorecen la inclusión y cohesión social (Valls et. al, 2014).
} 
ReVista de La Escuela de CIENCIAS DE LA EduCACIÓN, AÑo 17, NRO. 16, VOL. 2, JULIO A DiCIEMBRE DE 2021. PÁGINAS 135-144. ISSN 2362-3349 (EN LÍNEA). COMUNIDADES DE APRENDIZAJE: UNA EXPERIENCIA EN Y PARA LA PROFESIONALIZACIÓN DOCENTE EN LA UNIVERSIDAD NACIONAL DE EDUCACIÓNECUADOR. VÍCTOR Miguel SUMBA ARÉVALO. JOHANNA GRACE MEJÍA VERA.

El trabajo colaborativo entre docentes en la Comunidad de Aprendizaje constituye una estrategia que permite fortalecer la práctica pedagógica ya que, mediante la experiencia se identifican las fortalezas y debilidades. Como primer aspecto que conduce a la gestión del conocimiento constituye la participación voluntaria y activa en las comunidades de aprendizaje.

En la Comunidad de Aprendizaje compartí mi experiencia, que fortaleció a otros docentes sobre la comprensión lectora, demostrando la participación activa, voluntaria y responsable. Así mismo aceptar de otras personas, que enriquecieron mis conocimientos, que me ayudaron a reflexionar lo que me falta por aprender, motivando a seguir investigando y actualizando diariamente (Martha, docente participante en la CA).

Es de esta manera, cómo la docente en cuestión atribuye valor tanto a sus propios conocimientos como a los conocimientos que posee el otro. Esto le asigna importancia a la participación de los docentes de manera responsable, en ello radica el propio interés por pertenecer al grupo e investigar para aprender.

La visión de los participantes con relación al trabajo desarrollado mediante la cooperación indica que "compartir experiencias entre docentes ha permitido enriquecer nuestros conocimientos, porque trabajamos de forma voluntaria, activa, cooperativa y equitativa, donde de una manera reflexiva permitió corregir nuestras falencias pedagógicas" (Grupo de discusión). En tal sentido, otro punto clave para la gestión del conocimiento en la Comunidad de Aprendizaje se da mediante la relación recíproca y la cooperación en ella. Dan importancia a lo que cada uno de los participantes manifiesta en relación con la comprensión de la problemática.

También permite poner de manifiesto la relación o no de las prácticas que se han desarrollado durante la carrera docente con la revisión teórica que las fundamenta. Al desarrollar la revisión documental sobre la problemática, en su mayoría relacionadas con las estrategias didácticas, evidencian la coherencia entre los resultados e información encontrada y lo que verdaderamente realizan en el aula. "Al realizar el estado del arte comprendí las diferentes fases para desarrollar la comprensión lectora, en mi práctica he desarrollado algo similar, pero con la investigación sé lo que tengo que hacer (Pedro, docente participante)". Es así como un nuevo proceso de gestión es evidente en el proceso de formación, la revisión documental.

Dicha indagación se relaciona con otro proceso de gestión que hemos identificado y a lo que los docentes determinan como un proceso de reflexión; es decir, detenerse un momento y revisar, en este caso dialogar sobre la propia práctica.

El objetivo ha sido de reflexionar nuestra propia práctica docente, desaprendiendo lo tradicional y reaprendiendo nuevos conocimientos, con esto me di cuenta que existen otras maneras de aprender. Con el diálogo igualitario y trabajo colaborativo entre los docentes. (Martha, docente participante).

De igual manera, otra docente manifiesta:

Esta metodología de trabajo me ha permitido reflexionar sobre mi práctica docente, la misma que he tomado no como una problemática, sino más bien una oportunidad para fortalecer, mejorar y analizar los logros alcanzados, además mantener un diálogo constante con todos los miembros de la Comunidad de Aprendizaje (Elsa, docente participante).

En las citas anteriores se pueden observar, además, nuevos términos como desaprender y reaprender, mismos que constituyen nuevos significados y existe una aprehensión de los docentes al relacionarlos directamente con el proceso de nuevos aprendizajes en las comunidades.

La participación igualitaria de todos los docentes es fundamental para consolidar la comunidad y generar nuevos aprendizajes. El diálogo igualitario hace énfasis en los argumentos más que en la posición jerárquica de los participantes, del ambiente emocional en la comunidad dependerá la participación de los docentes. Uno de los docentes, en la reflexión sobre su participación en la Comunidad de Aprendizaje manifiesta que el diálogo igualitario:

permite enriquecer nuestros conocimientos y que serán puestos en práctica en nuestro quehacer docente, fomentando que todos aprendemos de todos. En tal virtud los aportes de cada uno de los actores que se encuentran sumergidos ante este cambio, nos facilitan a identificar las problemáticas que son causantes en el fortalecimiento de una convivencia armónica del proceso de enseñanza aprendizaje (Elsa, docente participante).

La formación dialógica del profesorado implementado en las Comunidades de Aprendizajes constituye una estrategia que fortalece el aprendizaje colaborativo entre docentes. A través del diálogo entre docentes se inicia la fundamentación del quehacer pedagógico. En este sentido se encuentra en esta estrategia la posibilidad de reflexionar de forma conjunta sobre las estrategias aplicadas y conocer nuevas formas de proponer un aprendizaje participativo y significativo. Sin embargo, también se ha observado durante el diagnóstico y la ejecución del aprendizaje dialógico del profesorado que ciertos miembros se limitan a escuchar a los demás o intervienen en ciertas ocasiones, pero no se podría negar la influencia de lo escuchado en las formas de comprender y relacionar con su práctica. 
ReVista de La Escuela de CIENCIAS DE LA EduCACIÓN, AÑo 17, NRO. 16, VOL. 2, JULIO A DiCIEMBRE DE 2021. PÁGINAS 135-144. ISSN 2362-3349 (EN LÍNEA). COMUNIDADES DE APRENDIZAJE: UNA EXPERIENCIA EN Y PARA LA PROFESIONALIZACIÓN DOCENTE EN LA UNIVERSIDAD NACIONAL DE EDUCACIÓNECUADOR. VÍCTOR MIGUEL SUMBA ARÉVALO. JOHANNA GRACE MEJÍA VERA.

Los datos generados durante el proceso son sistematizados en el PIENSA con diferentes términos pero con el mismo significado. Así, los docentes exponen que:

La organización entre profesores nos ha permitido conformar la CPA, como una forma de evaluar las reflexiones de nuestras experiencias de la práctica docente, ha permitido fortalecer los conocimientos del profesorado, recopilando estrategias activas para la comprensión lectora, para poner en práctica con los estudiantes a lo posterior (PIENSA del Grupo 1).

\section{Otro grupo expresa;}

Se asumen responsabilidades por parte de los docentes para mejorar sus conocimientos y a través de las nuevas estrategias de compresión mejorar su proceso de enseñanza. Se vislumbra el optimismo para sacar adelante sus enseñanzas a los estudiantes y ese compromiso en seguir formando parte de la CPA (PIENSA del grupo 2).

De lo presentado anteriormente, con relación a la participación docente y la gestión de nuevos conocimientos se puede interpretar el valor que otorgan los docentes a las Comunidades de Aprendizajes como espacios que contribuyen a mejorar su quehacer docente. Así pueden identificarse expresiones como "La CPA también permite ver debilidades y fortalezas de nuestra práctica diaria, una comunidad de aprendizaje debe caracterizarse en el que todos tienen algo que aprender y algo que enseñar [...] además, permite crear espacios para construir nuevos conocimientos" (PIENSA de grupo 3). También nos lleva a identificar la necesidad de ampliar la temporalidad en la intervención en las Comunidades de Aprendizaje para consolidar aprendizajes, dicha necesidad también lo explicitan los docentes "muchas de las veces los resultados no se reflejarán de noche a la mañana, pero estamos seguros que, con el trabajo mancomunado y consensuado de los integrantes de las Comunidades de Aprendizaje, obtendremos cambios positivos en nuestra labor docente" (PIENSA grupo 4).

Por otro lado, el análisis y reflexión de la práctica docente se convierte en el eje transversal en las Comunidades de Aprendizaje, los estudiantes-docentes (y todos los integrantes) ponen de manifiesto las necesidades e intereses en la fase diagnóstico, lo que necesariamente involucra volver la mirada al quehacer del docente. Esta reflexión se complementa con el contraste teórico y la propia práctica, lo que lleva a fortalecer o modificarla de acuerdo a los resultados de investigaciones; "Al realizar el estado del arte comprendí las diferentes fases para desarrollar la comprensión lectora, en mi práctica he desarrollado algo similar, pero con la investigación sé lo que tengo que hacer (Pedro, docente participante)" A esto se suma el proceso dialógico, en el cual cada participante comparte experiencias y relaciona con lo realizado por otro docente. En este sentido otorga valor al conocimiento experiencial de otros docentes y al conocimiento construido desde las investigaciones.

En definitiva, a través de la participación de los estudiantes-docentes en las Comunidades de Aprendizaje como parte de su formación en la UNAE se han constituido espacios que permiten la interacción con los otros y el intercambio de conocimientos teóricos y prácticos desde la propia experiencia docente. Las actividades desarrolladas en la ejecución de dichas comunidades han permitido identificar la actuación de éstos en la gestión de nuevos conocimientos o en la resignificación de las prácticas ya desarrolladas por ellos. En tal sentido, la propuesta de las Comunidades de Aprendizaje comprende espacios de debate, reflexión, reconceptualización, desaprendizajes y reaprendizajes en y para la profesionalización docente.

\section{Referencias bibliográficas}

Alcedo, A.; Chacón, C. y Chacón, M. (2014). Las Comunidades de Aprendizaje: estrategia de desarrollo profesional de los docentes de inglés. Educere, 18(61), pp.483-494. https://www.redalyc.org/pdf/356/35639776010.pdf

Alen, B. (2013). Los ateneos: un dispositivo de acompañamiento a los docentes noveles en Alen, B., Alliaud, A., Hevia, R., Ramírez, J. y Castellano, R. (Ed.), Desarrollo profesional de formadores para el acompañamiento

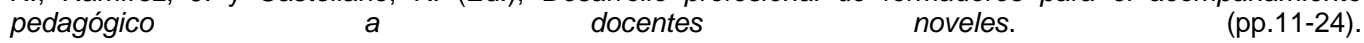
https://www.oei.es/historico/formaciondocente/materiales/OEI/URUGUAY_2012.pdf

Álvarez, C.; González, L., y Larrinaga, A. (2013). Aprendizaje dialógico: una apuesta de centro educativo para la inclusión. Tabanque: revista pedagógica, r (26), p.209-224. https://uvadoc.uva.es/bitstream/handle/10324/11986/Tabanque-2013-26 AprendizajeDialogicoUnaApuestaDeCentroEducativoPar.pdf?sequence $=1$

Assael, J. y Guzmán, I. (1996). Procesos grupales y aprendizaje en talleres de educadores: una propuesta para la formación de docentes. Revista Colombiana de Educación, (331), pp.11-15. https://revistas.pedagogica.edu.co/index.php/RCE/article/view/5399

Aubert, A.; García, C. y Racionero, S. (2009). El aprendizaje dialógico. Cultura y educación, 21(2), pp.129-139. https://www.viaeducacion.org/downloads/ap/ehd/aprendizaje_dialogico.pdf

Beltrán, Y.; Martínez, Y. y Torrado, O. (2015). Creación de una comunidad de aprendizaje: una experiencia de educación inclusiva en Colombia. Revista Encuentros, Universidad Autónoma del Caribe, 13(2), pp.57-72. http://www.scielo.org.co/pdf/encu/v13n2/v13n2a04.pdf

Botton, L. (2015). La dimensión instrumental en las comunidades de aprendizaje. Intangible Capital, 11(3), pp.305-371. https://www.redalyc.org/pdf/549/54941394005.pdf

Cadena, M. y Orcasitas, J. (2016). Comunidades de Aprendizaje en el País Vasco: caracterización y organización escolar. Educación y Educadores, 19(3), pp.373-391. https://www.redalyc.org/pdf/834/83448566004.pdf 
REVISTA DE LA ESCUELA DE CIENCIAS DE LA EduCACIÓN, AÑo 17, NRO. 16, VOL. 2, JULIO A DICIEMBRE DE 2021. PÁGINAS 135-144. ISSN 2362-3349 (EN LÍNEA). COMUNIDADES DE APRENDIZAJE: UNA EXPERIENCIA EN Y PARA LA PROFESIONALIZACIÓN DOCENTE EN LA UNIVERSIDAD NACIONAL DE EDUCACIÓNECUADOR. VÍCTOR Miguel SUMBA ARÉVALO. JOHANNA GRACE MEJÍA VERA.

Cantero, N. y Pantoja, A. (2016). La transformación de centros educativos en comunidades de aprendizaje (CdA) en la provincia de Jaén. Profesorado. Revista de Currículum y Formación de Profesorado, 20(3), pp.715-749. https://www.redalyc.org/pdf/567/56749100021.pdf

Castro, M.; Gómez, A. y Macazaga, A. (2014). Aprendizaje dialógico y grupos interactivos en educación física. Retos: nuevas tendencias en educación física, deporte y recreación, 25(1), pp.174-179. https://dialnet.unirioja.es/servlet/articulo?codigo $=4555265$

CIPPEC. (2018, mayo 14). CdeA - Formación dialógica del profesorado [Archivo de video]. https://www.youtube.com/watch?v=RE ED6WdmAM

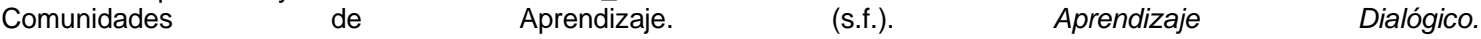
https://comunidadesdeaprendizaje.net/presentacion/aprendizaje-dialogico/

De Antonio, M. y Gonçalves, D. (2017). Aprendizajes situados y desarrollo de cultura democratizadora en el marco de las culturas Institucionales. Dedica. Revista de Educação e Humanidades, (11), pp.25-46. https://dialnet.unirioja.es/servlet/articulo?codigo $=5906266$

Domínguez, F. (2018). Fundamentos y características de un modelo inclusivo y de calidad educativa: Comunidades de

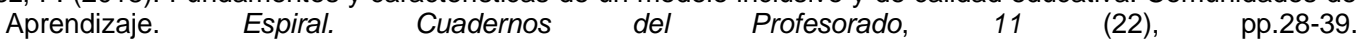
http://repositorio.ual.es/bitstream/handle/10835/5874/1915-5523-1-PB.pdf?sequence=1

Flecha, R. [GNOSS]. (2011, abril 27). Comunidades de Aprendizaje: entrevista a Ramón Flecha [Archivo de video]. https://www.youtube.com/watch?v=Rs7 XSNKehA

García, J. y Giacobbe, M. (2009). Nuevos desafíos en investigación. Argentina: Homo Sapiens.

Herrera, J. y Guevara, G. (2019). Las estrategias organizativas y metodológicas para la atención a la diversidad en el aula: innovar para enseñar. En: Bello, D. y Guillén, G. Educación inclusiva, un debate necesario (37-65). UNAE EP https://www.oei.es/historico/formaciondocente/materiales/OEI/URUGUAY_2012.pdf\#page=11

Jiménez, A. y Rodríguez, M. (2016). Comunidades de Aprendizaje: propuesta de desarrollo y sostenibilidad desde la educación social en instituciones educativas. Cuestiones Pedagógicas, 25(1), pp.105-118. http://institucional.us.es/revistas/cuestiones/25/08_Ml_25.pdf

Krichesky, G. y Torrecilla, F. (2011). Las Comunidades Profesionales de Aprendizaje: Una estrategia de mejora para una nueva concepción de escuela. REICE: Revista Iberoamericana sobre Calidad, Eficacia y Cambio en Educación, 9(1), 65-83. https://dialnet.unirioja.es/descarga/articulo/3934351.pdf

Krichesky, J. y Murillo, J. (2011). Las Comunidades Profesionales de Aprendizaje una estrategia de mejora para una nueva concepción de escuela, Revista Iberoamericana sobre Calidad, Eficacia y Cambio en Educación, 9(1), 65-83. http://www.rinace.net/reice/numeros/arts/vol9num1/art4.pdf

Molina, S. (2015). La inclusión del alumnado con necesidades educativas. OmniaScience, 11(3): pp.372-392. https://core.ac.uk/download/pdf/41825005.pdf

Morales, L., Chaclán, C., Maldonado, S., Sontay, G., Montenegro, R. y Magzu,I J. (2013). Las Comunidades de Aprendizaje $\quad y \quad$ círculos $\quad d e$

Pérez, A. (2012). Educarse en la era digital. Madrid: Morata.

Roca, E. (2016). Formación dialógica del profesorado, reencanto con la profesión docente. Comillas, 367, pp.11-16. https://revistas.comillas.edu/index.php/padresymaestros/article/viewFile/7116/6949

Salamanca, Y.; Chacón, C. y Corzo, M. (2014). Las Comunidades de Aprendizaje: estrategia de desarrollo profesional de los docentes de inglés. Educere, 18(61), 483-494. https://www.redalyc.org/pdf/356/35639776010.pdf

Soria, M. y Hernández, R. (2017). Aportes a la formación docente desde comunidades de aprendizaje. Revista del Cisen Tramas/Maepova, 5(2), pp.131-147. http://www.academia.edu/download/58152569/TRAMAS_9.10.pdf

Torres, R. (2004). Comunidad de Aprendizaje Repensando lo educativo desde el desarrollo local y el aprendizaje. http://200.10.23.169/educacion/aprendizaje_vida_comunidad_aprendizaje_esp.pdf

Trujillo, J.; Aznar, I. y Cáceres, M. (2015). Análisis del uso e integración de redes sociales colaborativas en Comunidades de Aprendizaje de la Universidad de Granada (España) y John Moores de Liverpool (Reino Unido). Revista Complutense de Educación, 26(1), pp.289-311. https://core.ac.uk/download/pdf/38821565.pdf

Universidad Nacional de Educación. (2017). Modelo Pedagógico de la UNAE. https://unae.edu.ec/wpcontent/uploads/2019/11/modelo-pedagogico-unae.pdf

Valls, R. y Munté, A. (2010). Las claves del aprendizaje dialógico en las Comunidades de Aprendizaje. Profesorado. Revista Interuniversitaria de Formación del Profesorado, 24(1), pp.11-15. https://www.redalyc.org/articulo.oa?id=274/27419180001

Valls, R.; Prados, M. y Aguilera, A. (2014). El proyecto INCLUD-ED: estrategias para la inclusión y la cohesión social en Europa desde la educación. Revista de Investigación e Innovación Educativa, 82(2), pp.31-43. https://revistascientificas.us.es/index.php/IE/article/view/6885

Vilanova, A.; Pérez, P., Durán, E. y Mendieta, C. (2017). Licenciatura en Educación Intercultural Bilingüe. Modalidad a distancia (Proyecto Innovador de Carrera). Material no publicado. 